\title{
A Fast and Parallel Stroud-Based Stochastic Collocation Method for Statistical EMI/EMC Analysis
}

\author{
Hakan Bağc1 \\ Department of \\ Electrical Engineering, \\ University of \\ Michigan, Ann Arbor, \\ MI 48109, USA
}

\author{
Çağlar Yavuz \\ Department of \\ Electrical Engineering, \\ University of \\ Michigan, Ann Arbor, \\ MI 48109, USA
}

\author{
Abdülkadir C. Yücel \\ Department of \\ Electrical Engineering, \\ University of \\ Michigan, Ann Arbor, \\ MI 48109, USA
}

\author{
Jan S. Hesthaven \\ Division of Applied \\ Mathematics, Brown \\ University, \\ Providence, RI \\ 02912, USA
}

\author{
Eric Michielssen \\ Department of \\ Electrical Engineering, \\ University of \\ Michigan, Ann Arbor, \\ MI 48109, USA
}

\begin{abstract}
A fast and parallel Stroud-based stochastic collocation method for statistically characterizing electromagnetic interference and compatibility (EMI/EMC) phenomena on loaded multiscale platforms with uncertain system configurations and subject to variable electromagnetic excitations is described. The proposed method uses a previously developed hybrid time domain integral equation based field-cable-circuit to carry out deterministic EMI/EMC simulations permitting the statistical characterization of pertinent observables. The number of simulations required by the proposed method is far fewer than those needed by Monte-Carlo methods. The proposed method is used to characterize cable-induced coupling onto PC cards located in shielding enclosures. Both the hybrid simulator and the stochastic collocation code execute with near-full efficiency on distributed memory clusters.
\end{abstract}

Keywords - electromagnetic interference and compatibility, stochastic collocation, Stroud integration rules, electromagnetic coupling, time domain integral equations, hybrid solvers, fast solvers, parallelization

\section{INTRODUCTION}

Modern vehicles come replete with electronic communication and navigation systems fed and interconnected by imperfectly shielded cables. Electromagnetic simulators are needed to characterize these systems' vulnerability to internally and externally generated electromagnetic interference. For these simulators to be fully useful, they must be computationally efficient and offer multiscale simulation capabilities to permit the analysis of electromagnetic phenomena on electrically large platforms loaded with (sub)wavelength-scale systems. Furthermore, they must be able to accurately account for uncertainty in the system configuration (viz. placement of the electronic systems and cables, parameterization of device and circuit descriptions, etc.), and the electromagnetic excitation. This task is complicated by the fact that electromagnetic fields inside the electrically large platform often behave quasi-chaotically. Small perturbations in the placement of the electronic systems and especially cables, which invariably is subject to installation uncertainty, may dramatically impact the electromagnetic interference and compatibility (EMI/EMC) performance of the overall system.

The fast Fourier transform (FFT)-accelerated parallel hybrid time domain integral equation (TDIE)-based fieldcable-circuit simulator described in [1-6] permits the efficient EMI/EMC analysis of deterministically configured, electrically large, and multiscale platforms. This simulator has successfully addressed the first of the above mentioned two simulation challenges. To address second simulation challenge, we used this simulator in conjunction with a parallel implementation of the stochastic collocation method described in [7]. This approach to statistically characterize EMI/EMC problems requires far fewer function evaluations (i.e., deterministic EMI/EMC simulations) than the MonteCarlo methods that were previously used for this purpose. The method achieves this efficiency by evaluating the quantity to be characterized, e.g. a voltage observed deep into an electronic system, at carefully chosen collocation points in the space of system configurations and electromagnetic excitations. These collocation points stem from basic Stroud-2 and Stroud-3 numerical integration rules [8] that are easily modified to account for the presence of a probability density/weighting function. Given $N$ variable input parameters, these integration rules call for the evaluation of the quantity to be characterized at $N+1$ or $2 N$ cubature points.

\section{FORMULATION}

\section{A. Description of the Deterministic Simulator}

This section describes the deterministic hybrid field-cablecircuit simulator and details its three solver components as well as their simultaneous coupling. 
TDIE-Based Field Solver: A standard TDIE [5] is used to describe external field interactions. The surface of the exterior structure is represented by perfect electrically conducting bodies and thin wires, which may be attached to the bodies by means of junctions. The current density on this surface is expanded using $N_{\mathrm{EM}}$ spatial and $N_{t}$ temporal basis functions. Then, this expansion is inserted into the TDIE and the resulting equation is tested using Galerkin's method in space and point matching in time. This results in a $N_{\mathrm{EM}} \times N_{\mathrm{EM}}$ linear system of equations at time $t=l \Delta t, l=1, \ldots, N_{\mathrm{t}}$, where $\Delta t$ is the time step size:

$$
\mathbf{Z}_{0}^{\mathrm{EM}} \mathbf{I}_{l}^{\mathrm{EM}}=\mathbf{V}_{l}^{\mathrm{ei}}+\mathbf{V}_{l}^{\mathrm{ec}}-\sum_{l^{\prime}=1}^{l-1} \mathbf{Z}_{l-l^{\prime}}^{\mathrm{EM}} \mathbf{I}_{l^{\prime}}^{\mathrm{EM}} .
$$

Here, $\mathbf{I}_{l}^{\mathrm{EM}}$ is the vector of unknown current coefficients, $\mathbf{Z}_{l-l^{\prime}}^{\mathrm{EM}}$ are impedance matrices, and $\mathbf{V}_{l}^{\mathrm{ei}} / \mathbf{V}_{l}^{\mathrm{ec}}$ is the vector of tested incident fields due to impressed/controlled sources, which represents external excitations/coupling from connectors/loads.

TDIE-Based Transmission Line Solver: Standard transmission-line equations [9] are used to describe guidedfield interactions. Time varying coefficients of the current solution's homogeneous part on $N_{\mathrm{CBL}}$ cables are expanded using $N_{t}$ temporal basis functions. Then this expansion is inserted into the TDIEs enforced at the cable terminations and the resulting equations are tested using point matching in time. This results in a two $2 N_{\mathrm{CBL}} \times 2 N_{\mathrm{CBL}}$ linear systems of equations at time $t=l \Delta t, l=1, \ldots, N_{\mathrm{t}}$ :

$$
\begin{gathered}
\mathbf{I}_{l}^{\mathrm{te}}+\mathbf{I}_{l}^{\mathrm{h}}=\mathbf{I}_{l}^{\mathrm{ends}} \\
\mathbf{V}_{l}^{\mathrm{te}}+\mathbf{V}_{l}^{\mathrm{h}}=\mathbf{V}_{l}^{\mathrm{ends}} .
\end{gathered}
$$

Here, $\mathbf{I}_{l}^{\text {ends }}, \mathbf{I}_{l}^{\mathrm{te}}$, and $\mathbf{I}_{l}^{\mathrm{h}}$ are the vectors of total current, particular current, and homogenous current solution samples and $\mathbf{V}_{l}^{\text {ends }}, \mathbf{V}_{l}^{\text {te }}$, and $\mathbf{V}_{l}^{\mathrm{h}}$, are the vectors of total voltage, particular voltage, and homogenous voltage solution samples at the cable terminations, respectively. The vectors $\mathbf{I}_{l}^{\text {te }}$ and $\mathbf{V}_{l}^{\text {te }}$ are samples of current and voltage induced at the cable terminations due to $N_{\mathrm{CP}}$ series voltage and $N_{\mathrm{CP}}$ shunt current sources located along the transmission line that represent coupling from external fields, whereas the vectors $\mathbf{I}_{l}^{\mathrm{h}}$ and $\mathbf{V}_{l}^{\mathrm{h}}$ are related to the vector of unknown current coefficients $\mathbf{I}_{l}^{\mathrm{CBL}}$ through discrete convolutions:

$$
\mathbf{I}_{l}^{\mathrm{h}}=\sum_{l^{\prime}=1}^{l} \mathbf{G}_{l-l^{\prime}} \mathbf{I}_{l^{\prime}}^{\mathrm{CBL}}, \mathbf{V}_{l}^{\mathrm{h}}=\sum_{l^{\prime}=1}^{l} \mathbf{G}_{l-l^{\prime}}^{\mathrm{i}} \mathbf{I}_{l^{\prime}}^{\mathrm{CBL}} .
$$

Here, $\mathbf{G}_{l-l^{\prime}}$ and $\mathbf{G}_{l-l^{\prime}}^{\mathrm{i}}$ are propagation matrices. As the boundary conditions at the cable terminations are enforced by the circuit solver, which supplies $\mathbf{V}_{l}^{\text {ends }}$ at each time step, only (3) [and not (2)] is solved for $\mathbf{I}_{l}^{\mathrm{CBL}}$ :

$$
\mathbf{G}_{0}^{\mathrm{i}} \mathbf{I}_{l}^{\mathrm{CBL}}=\mathbf{V}_{l}^{\mathrm{ends}}-\mathbf{V}_{l}^{\mathrm{te}}-\sum_{l^{\prime}=1}^{l-1} \mathbf{G}_{l-l^{\mathrm{i}}}^{\mathrm{i}} \mathbf{I}_{l^{\prime}}^{\mathrm{CBL}} .
$$

MNA-Based Circuit Solver: Electrically small connecters/loads are represented by equivalent linear circuits. Enforcing Kirchhoff's equations for $N_{\text {CKT }}$ total number of non-ground nodes via MNA yields $N_{\text {CKT }} \times N_{\text {CKT }}$ linear system of equations at time $t=l \Delta t$ :

$$
\mathbf{Y}^{\mathrm{CKT}} \mathbf{V}_{l}^{\mathrm{CKT}}=\mathbf{I}_{l}^{\mathrm{CKT}, \mathrm{inc}}=\mathbf{I}_{l}^{\mathrm{ci}}+\mathbf{I}_{l}^{\mathrm{ce}}+\mathbf{I}_{l}^{\mathrm{ct}} \quad \text { for } l=1, \ldots, N_{\mathrm{t}} .
$$

Here, $\mathbf{V}_{l}^{\mathrm{CKT}}$ is the vector of unknown node voltages, $\mathbf{Y}$ is an admittance matrix, and $\mathbf{I}_{l}^{\mathrm{ci}}$ is the vector of impressed sources representing generators; $\mathbf{I}_{l}^{\mathrm{ce}} / \mathbf{I}_{l}^{\mathrm{ct}}$ is the vector of controlled source that represents coupling from external/guided fields.

\section{Coupled System of Equations}

I. Interfacing of Field and Circuit Solvers: To interface field and circuit solvers $\mathbf{I}_{l}^{\mathrm{ce}}$ and $\mathbf{V}_{l}^{\mathrm{ec}}$ are used:

$$
\mathbf{I}_{l}^{\mathrm{ce}}=\mathbf{C}^{\mathrm{ec} \dagger} \mathbf{I}_{l}^{\mathrm{EM}}, \mathbf{V}_{l}^{\mathrm{ec}}=-\sum_{l^{\prime}=l-3}^{l} \zeta_{l-l^{\prime}} \mathbf{C}^{\mathrm{ec}} \mathbf{V}_{l}^{\mathrm{CKT}}
$$

Here, the $N_{\mathrm{EM}} \times N_{\mathrm{CKT}}$ coupling matrix $\mathbf{C}^{\mathrm{ec}}$ selects the voltages at circuit terminals connected to the exterior system, the $N_{\mathrm{CKT}} \times N_{\mathrm{EM}}$ transposed matrix $\mathbf{C}^{\mathrm{ec} \dagger}$ selects the coefficients of spatial basis functions connected to circuit terminals, and the scalars $\zeta_{l-l^{\prime}}$ are the coefficients of a thirdorder backward differentiation formula that is used to compute the temporal derivative of the coupled fields as required by the field solver's TDIE.

II. Interfacing of Field and Transmission-line Solvers: To interface field and transmission-line solvers $\mathbf{I}_{l}^{\text {te }}$ and $\mathbf{V}_{l}^{\text {te }}$ are used:

$$
\begin{aligned}
& \mathbf{I}_{l}^{\mathrm{te}}=0.5\left(\sum_{\tilde{l}=1}^{l} \mathbf{S}_{l-\tilde{l}}^{\nabla} \mathbf{J}_{\tilde{l}}^{\mathrm{te}}+\sum_{\tilde{l}=1}^{l} \mathbf{S}_{l-\tilde{l}}^{\mathrm{a}} \mathbf{E}_{\tilde{l}}^{\mathrm{te}}\right), \\
& \mathbf{V}_{l}^{\mathrm{te}}=0.5\left(\sum_{\tilde{l}=1}^{l} \mathbf{S}_{l-\bar{l}}^{\mathrm{i}, \nabla} \mathbf{J}_{\tilde{l}}^{\mathrm{te}}+\sum_{\tilde{l}=1}^{l} \mathbf{S}_{l-\tilde{l}} \mathbf{E}_{\tilde{l}}^{\mathrm{te}}\right) .
\end{aligned}
$$

Here the $2 N_{\mathrm{CBL}} \times N_{\mathrm{CP}}$ propagation matrices $\mathbf{S}_{l-\tilde{l}}^{\nabla}, \mathbf{S}_{l-\tilde{l}}^{\mathrm{a}}$, $\mathbf{S}_{l-\tilde{l}}^{\mathrm{i}, \nabla}$, and $\mathbf{S}_{l-\tilde{l}}$ relate $\mathbf{I}_{l}^{\text {te }}$ and $\mathbf{V}_{l}^{\text {te }}$ to the $\mathbf{J}_{\tilde{l}}^{\text {te }}$ and $\mathbf{E}_{\tilde{l}}^{\text {te }}$ (samples of sources located along the transmission line that represent coupling from external fields). These samples are in turn related to $\mathbf{I}_{l^{\prime}}^{\mathrm{EM}}$ :

$$
\mathbf{J}_{\tilde{l}}^{\mathrm{te}}=2 \pi a \sum_{l^{\prime}=1}^{\tilde{l}} \mathbf{T}_{\tilde{l}-l^{\prime}}^{\mathrm{a}} \mathbf{P}^{\mathrm{te}} \mathbf{I}_{l^{\prime}}^{\mathrm{EM}}, \mathbf{E}_{\tilde{l}}^{\mathrm{te}}=2 \pi a \sum_{l^{\prime}=1}^{\tilde{l}} \mathbf{T}_{\tilde{l}-l^{\prime}}^{\mathrm{i}} \mathbf{P}^{\mathrm{te}} \mathbf{I}_{l^{\prime}}^{\mathrm{EM}} .
$$

Here, the $N_{\mathrm{CP}} \times N_{\mathrm{EM}}$ permutation matrix $\mathbf{P}^{\text {te }}$ selects the $N_{\text {CP }}$ current coefficients that correspond to the space-time basis functions located on shields of cables, $N_{\mathrm{CP}} \times N_{\mathrm{CP}}$ diagonal coupling matrices $\mathbf{T}_{\tilde{l}-l^{\prime}}^{\mathrm{a}}$ and $\mathbf{T}_{\tilde{l}-l^{\prime}}^{\mathrm{i}}$, which are obtained 
by discretizing transfer impedance and admittance functions, relate these coefficients to $\mathbf{J}_{\tilde{l}}^{\text {te }}$ and $\mathbf{E}_{\tilde{l}}^{\text {te }}$.

III. Interfacing of Transmission-Line and Circuit Solvers: To interface transmission-line and circuit solvers, current and voltage vectors $\mathbf{I}_{l}^{\mathrm{ct}}$ and $\mathbf{V}_{l}^{\mathrm{ends}}$ are used:

$$
\mathbf{I}_{l}^{\mathrm{ct}}=\mathbf{C}^{\mathrm{tc} \dagger} \mathbf{I}_{l}^{\mathrm{ends}}=\mathbf{C}^{\mathrm{tc} \dagger}\left(\mathbf{I}_{l}^{\mathrm{te}}+\mathbf{I}_{l}^{\mathrm{h}}\right), \mathbf{V}_{l}^{\mathrm{ends}}=-\mathbf{C}^{\mathrm{tc}} \mathbf{V}_{l}^{\mathrm{CKT}} .
$$

Here the $2 N_{\mathrm{CBL}} \times N_{\mathrm{CKT}}$ coupling matrix $\mathbf{C}^{\text {tc }}$ selects the voltages at circuit terminals connected to the cable terminations and the transposed matrix $\mathbf{C}^{\mathrm{tc}^{\dagger} \dagger}$ selects the transmission-line currents at the cable terminations connected to the circuit terminals.

VI. Final System of Equations: All three solvers are coupled by combining (1), (5), and (6), and substituting (7) for $\mathbf{I}_{l}^{\mathrm{ce}}$ and $\mathbf{V}_{l}^{\mathrm{ec}}$, (4) for $\mathbf{I}_{l}^{\mathrm{h}}$ and $\mathbf{V}_{l}^{\mathrm{h}},(8)$ for $\mathbf{I}_{l}^{\mathrm{te}}$ and $\mathbf{V}_{l}^{\mathrm{te}}$, and (10) for $\mathbf{I}_{l}^{\text {ct }}$ and $\mathbf{V}_{l}^{\text {ends }}$. Using (9) for $\mathbf{J}_{l}^{\mathrm{te}}$ and $\mathbf{E}_{l}^{\mathrm{te}}$ in the resulting equation, a linear system of equations of size $\left(N_{\mathrm{EM}}+N_{\mathrm{CKT}}+2 N_{\mathrm{CBL}}\right) \times\left(N_{\mathrm{EM}}+N_{\mathrm{CKT}}+2 N_{\mathrm{CBL}}\right)$ is obtained:

$$
\left[\begin{array}{ccc}
\mathbf{Z}_{0}^{\mathrm{EM}} & \zeta_{0} \mathbf{C}^{\mathrm{ec}} & 0 \\
-\mathbf{C}^{\mathrm{cte}}-\mathbf{C}^{\mathrm{ec} \dagger} & \mathbf{Y}^{\mathrm{CKT}} & \mathbf{C}^{\mathrm{ch}} \\
-\mathbf{C}^{\mathrm{te}} & \mathbf{C}^{\mathrm{tc}} & \mathbf{G}_{0}^{\mathrm{i}}
\end{array}\right]\left[\begin{array}{c}
\mathbf{I}_{l}^{\mathrm{EM}} \\
\mathbf{V}_{l}^{\mathrm{CKT}} \\
\mathbf{I}_{l}^{\mathrm{CBL}}
\end{array}\right]=\left[\begin{array}{c}
\mathbf{V}_{l}^{\mathrm{ei}}+\hat{\mathbf{V}}_{l}^{\mathrm{e}}+\hat{\mathbf{V}}_{l}^{\mathrm{ec}} \\
\mathbf{I}_{l}^{\mathrm{ci}}+\widehat{\mathbf{I}}_{l}^{\mathrm{cte}}+\widehat{\mathbf{I}}_{l}^{\mathrm{ch}} \\
\hat{\mathbf{V}}_{l}^{\mathrm{h}}+\hat{\mathbf{V}}_{l}^{\mathrm{te}}
\end{array}\right] .
$$

Here, the matrices $\mathbf{C}^{\text {cte }}, \mathbf{C}^{\text {ch }}$, and $\mathbf{C}^{\text {te }}$, and the vectors $\hat{\mathbf{V}}_{l}^{\text {ec }}, \quad \hat{\mathbf{I}}_{l}^{\text {cte }}, \quad \hat{\mathbf{I}}_{l}^{\text {ch }}$, and $\hat{\mathbf{V}}_{l}^{\text {te }}$ represent instantaneous and timedelayed coupling among the solvers, respectively, and the vectors $\hat{\mathbf{V}}_{l}^{\mathrm{e}}$ and $\hat{\mathbf{V}}_{l}^{\mathrm{h}}$ represent time-delayed interactions. Detailed expressions of these matrices and vector can be found in [1]. Equation (11) is solved simultaneously for all unknowns at each and every time step using an iterative solver. Matrices on the left-hand side of (11), $\mathbf{Z}_{0}^{\mathrm{EM}}, \mathbf{Y}^{\mathrm{CKT}}$, $\mathbf{G}_{0}^{\mathrm{i}}, \mathbf{C}^{\text {cte }}, \mathbf{C}^{\text {ch }}, \mathbf{C}^{\text {te }}, \mathbf{C}^{\mathrm{ec}}$, and $\mathbf{C}^{\text {tc }}$ are typically sparse or even diagonal. Hence the computational cost of solving (11) is dominated by the computation of convolutions required to evaluate the right-hand side vectors, $\left\{\hat{\mathbf{V}}_{l}^{\mathrm{h}}, \widehat{\mathbf{I}}_{l}^{\text {cte }}, \hat{\mathbf{I}}_{l}^{\text {ch }}, \widehat{\mathbf{V}}_{l}^{\text {te }}\right\}$ and $\hat{\mathbf{V}}_{l}^{\mathrm{e}}$, which require $O\left(N_{\mathrm{t}}^{2}\right)$ and $O\left(N_{\mathrm{t}} N_{\mathrm{EM}}^{2}\right)$, respectively. Using FFT-based acceleration schemes of $[5,6]$, these computational costs are reduced to $O\left(N_{\mathrm{t}} \log ^{2} N_{\mathrm{t}}\right)$ and $O\left(N_{\mathrm{t}} N_{\mathrm{c}} \log ^{2} N_{\mathrm{c}}\right)$, where $N_{\mathrm{c}} \sim N_{\mathrm{EM}}^{1.5}$ is the number of point sources located on an auxiliary Cartesian grid that encloses the exterior structure, respectively. The overall cost of the solution is further reduced by using parallel computation to $O\left(N_{t} N_{c} \log ^{2} N_{c} / P_{\mathrm{EM}}\right)$ operations per processor when $P_{\mathrm{EM}}$ processors are assigned to the field solver [4].

\section{B. Stroud-Based Stochastic Collocation Method}

Let $\mathbf{x}=\left[x^{1}, x^{2}, \ldots, x^{N-1}, x^{N}\right]$ be an $N$-dimensional variable, where each $x^{i}, i=1, \ldots, N$, represents a variable in the uncertain system configuration (such as placement of the electronic systems and cables, parameterization of device and circuit descriptions, etc.) and the excitation, and is uniformly distributed in interval $\left[b_{s}^{i}, b_{e}^{i}\right], i=1, \ldots, N$. As a measure of EMI/EMC characterization, average and standard deviation of $\tilde{V}(\mathbf{x})$, Fourier transform of the transient coupled voltage observed at the ports of an electronic system, will be computed. The deterministic hybrid simulator described above is used to compute $\tilde{V}(\mathbf{x})$ for a given $\mathbf{x}$ (Note that a Fourier transform is needed after the time-domain simulation is performed). To approximate the multivariable integrals required in the computation of the average and standard deviation of $\tilde{V}(\mathbf{x})$ higher order Stroud rules [8] are used. Assume that $\mathbf{z}=\left[z^{1}, z^{2}, \ldots, z^{N-1}, z^{N}\right]$ is an $N$-dimensional variable where each $z^{i}, i=1, \ldots, N$, is uniformly distributed in interval $[-1,1]$, and the integral

$$
I=\int_{(-1,1)^{N}} \tilde{V}(\mathbf{z}) d \mathbf{z}
$$

can only be evaluated numerically. Using the Stroud-2 and Stroud-3 numerical integration rules [8], (12) can be approximated as

$$
I \cong \sum_{i=1}^{M} w_{i} \tilde{V}\left(\mathbf{z}_{i}\right)
$$

Here $\mathbf{z}_{i}=\left[z_{i}^{1}, z_{i}^{2}, \ldots, z_{i}^{N-1}, z_{i}^{N}\right]$ are the cubature points and $w_{i}, i=1, \ldots, N+1$, are the weights. For Stroud-2, $M=N+1$, $w_{i}=2^{N} /(N+1), i=1, \ldots, N+1$, and $\mathbf{z}_{i}, i=1, \ldots, N+1$, are

$$
z_{i}^{2 k-1}=\sqrt{\frac{2}{3}} \cos \left(\frac{2 k(i-1) \pi}{N+1}\right), z_{i}^{2 k}=\sqrt{\frac{2}{3}} \sin \left(\frac{2 k(i-1) \pi}{N+1}\right),
$$

where $k=1, \ldots, \mid N / 2\rfloor$ and if $N$ is odd then $z_{i}^{N}=(-1)^{(i-1)} / \sqrt{3}$. For $\quad$ Stroud-3, $\quad M=2 N$, $w_{i}=2^{N} /(2 N), i=1, \ldots, N+1$, and $\mathbf{z}_{i}, i=1, \ldots, 2 N$, are

$$
z_{i}^{2 k-1}=\sqrt{\frac{2}{3}} \cos \left(\frac{(2 k-1) i \pi}{N}\right), z_{i}^{2 k}=\sqrt{\frac{2}{3}} \sin \left(\frac{(2 k-1) i \pi}{N}\right),
$$

where $k=1, \ldots,\lfloor N / 2\rfloor$ and if $N$ is odd then $z_{i}^{N}=(-1)^{i} / \sqrt{3}$. The expressions for the cubature points and the weights are derived assuming that the integral (12) is being computed in the range $[-1,1]$ in all $N$ dimensions. The linear mapping

$$
x_{i}^{k}=\frac{\left(z_{i}^{k}-1\right)\left(b_{e}^{k}-b_{s}^{k}\right)}{2}+b_{e}^{k}, i=1, \ldots, M, k=1, \ldots, N,
$$

is used to compute the locations of the integration points in each interval $\left[b_{s}^{k}, b_{e}^{k}\right], k=1, \ldots, N$. Additionally, weights of the numerical integration are scaled using

$$
\begin{aligned}
\widetilde{w}_{i} & =w_{i} \frac{\left(b_{e}^{1}-b_{s}^{1}\right) \cdots\left(b_{e}^{N}-b_{s}^{N}\right)}{2^{N}} \\
& =\frac{\left(b_{e}^{1}-b_{s}^{1}\right) \cdots\left(b_{e}^{N}-b_{s}^{N}\right)}{M}, i=1, \ldots, M .
\end{aligned}
$$

The average of $\tilde{V}(\mathbf{x})$ is defined as 


$$
E[\tilde{V}(\mathbf{x})]=\left(\prod_{k=1}^{N}\left(b_{e}^{k}-b_{s}^{k}\right)\right)^{-1} \int_{\left.\left[\left(s_{s}^{1}, b_{e} b^{1}\right) \ldots, \ldots, b_{s}^{N}, b_{e}^{N}\right)\right]} \tilde{V}(\mathbf{x}) d \mathbf{x} .
$$

Replacing the integral in (18) with (13) and using (16) and (17) in the resulting equation, one can obtain

$$
E[\tilde{V}(\mathbf{x})] \cong \frac{1}{M} \sum_{i=1}^{M} \tilde{V}\left(\mathbf{x}_{i}\right) .
$$

Standard deviation of $\tilde{V}(\mathbf{x})$ is defined as

$$
\begin{aligned}
\operatorname{dev}[\tilde{V}(\mathbf{x})] & =\left[\left(\prod_{k=1}^{N}\left(b_{e}^{k}-b_{s}^{k}\right)\right)^{-1} \int_{\left[\left(b_{s}^{1}, b_{e}^{1}\right), \ldots,\left(b_{s}^{N}, b_{e}^{N}\right)\right]} \tilde{V}^{2}(\mathbf{x}) d \mathbf{x}\right. \\
& \left.-(E[\tilde{V}(\mathbf{x})])^{2}\right]^{0.5} .
\end{aligned}
$$

Similarly, numerical evaluation of this integral can be done using

$$
\operatorname{dev}[\tilde{V}(\mathbf{x})] \cong\left[\frac{1}{M} \sum_{i=1}^{M} \tilde{V}^{2}\left(\mathbf{x}_{i}\right)-\left(\frac{1}{M} \sum_{i=1}^{M} \tilde{V}\left(\mathbf{x}_{i}\right)\right)^{2}\right]^{0.5}
$$

It should be noted here that Stroud-2 and Stroud-3 numerical integration rules require only $N+1$ and $2 N$

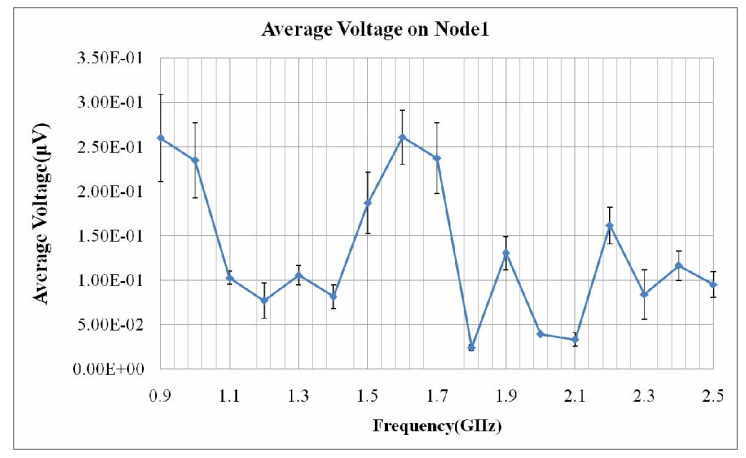

(a)

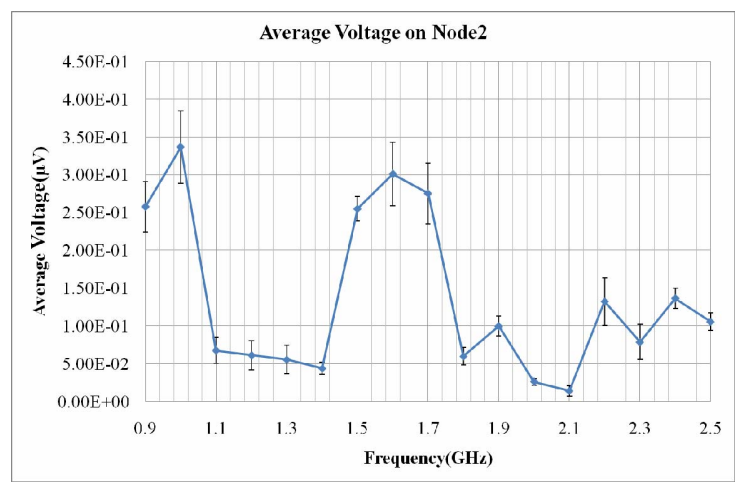

(b)

Figure 2 Average of the coupled voltages at (a) node 1 and (b) node 2 with error bars.

deterministic function evaluations (i.e. deterministic electromagnetic simulations), respectively. If a classical $P$ point numerical integration rule was used, $P^{N}$ deterministic function evaluations would be required.

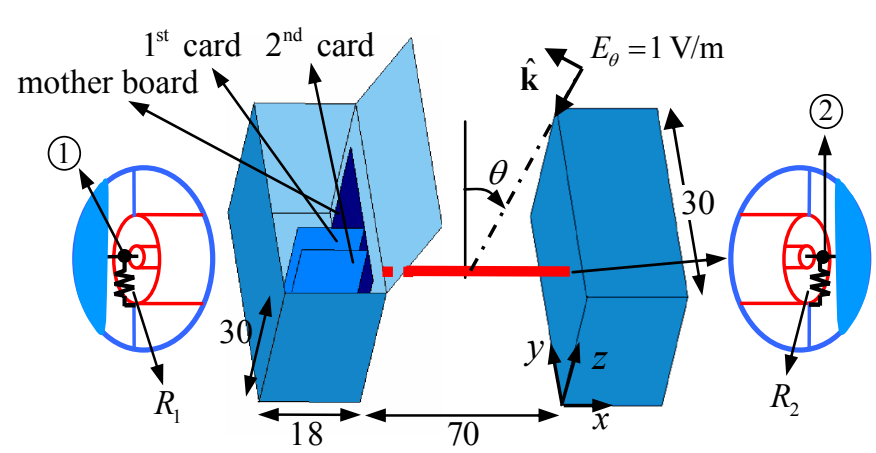

Figure1 PC cards, RG-58 cable connecting them, shielding enclosures, and plane-wave excitation.

\section{NUMERICAL ReSUltS}

The proposed method is used for estimating the average and standard deviation of coupled voltages at terminations of a RG-58 coaxial cable connecting two PC cards located inside shielding enclosures due to plane-wave excitation (Fig. 1). The RG-58 coaxial cable is filled with polyethylene dielectric filling and its outer shield radius $a_{\mathrm{o}}=1.524 \mathrm{~mm}$, inner shield radius $a_{\mathrm{i}}=1.397 \mathrm{~mm}$, and inner conductor radius $a=0.180 \mathrm{~mm}$. The shield's transfer impedance is given by

$\tilde{T}^{\mathrm{i}}(f)=R_{0}\left[(1+j)\left(a_{\mathrm{o}}-a_{\mathrm{i}}\right) / \delta\right] / \sinh \left[(1+j)\left(a_{\mathrm{o}}-a_{\mathrm{i}}\right) / \delta\right]+j 2 \pi f L_{a}$,

where $\delta=\sqrt{1 /\left(\pi f \sigma \mu_{0}\right)}$ is the skin depth, $f$ is the frequency, $\sigma=5.8 \times 10^{7} \mathrm{~S} / \mathrm{m}$ is the conductivity, $\mu_{0}$ is the free-space permeability, $R_{0}=14.3 \mathrm{~m} \Omega / \mathrm{m}, \quad$ and $L_{a}=1.0 \mathrm{nH} / \mathrm{m}$. The shielding enclosures are of identical size and they both contain a mother board and two daughter cards (Figs. 1). The two daughter cards are of identical size; the daughter card, which is closer to the back of the box ( $1^{\text {st }}$ card $)$, and the other one ( $2^{\text {nd }}$ card) are connected to the mother board with eight pins and one pin, respectively (See [1] for a more detailed description the cards and the pins). The RG-58 coaxial cable of length $70 \mathrm{~cm}$ connects the pins feeding the $2^{\text {nd }}$ cards. Two resistors, which are represented by $R_{1}$ and $R_{2}$ (Fig. 1) might potentially model the resistance of cable connectors, are also connected to feed pins. The current density on the surface of the shielding enclosures, the mother

Table I

\begin{tabular}{|c|c|c|}
\hline & node 1 & node2 \\
\hline Gauss: Average $(\mathrm{mV})$ & $0.260 \mathrm{E}-3$ & $0.258 \mathrm{E}-3$ \\
\hline Stroud: Average $(\mathrm{mV})$ & $0.260 \mathrm{E}-3$ & $0.260 \mathrm{E}-3$ \\
\hline Gauss: S. Deviation $(\mathrm{mV})$ & $0.481 \mathrm{E}-4$ & $0.324 \mathrm{E}-4$ \\
\hline Stroud: S. Deviation $(\mathrm{mV})$ & $0.490 \mathrm{E}-4$ & $0.333 \mathrm{E}-4$ \\
\hline
\end{tabular}

Comparison of the average and the standard deviation of the coupled voltages at nodes 1 and 2 at $\mathrm{f}=0.9 \mathrm{GHz}$ for Stroud-3 and 5-point Gauss-Legendre integration rules

boards, the daughter cards and the voltages on the loads are modeled by 21684 surface, 98 wire, and 120 junction basis 
functions, and 2 circuit unknowns, respectively. The number of transmission line unknowns is 2 . Coupling of the external and guided fields is realized at 76 points. The structure is illuminated by a plane wave propagating in $\hat{\mathbf{k}}=-\hat{\mathbf{x}} \sin (\theta)-\hat{\mathbf{z}} \cos (\theta)$ direction with $E_{\theta}=1 \mathrm{~V} / \mathrm{m}$ (Fig. 2). Using the Stroud-3 integration rule, the average and standard deviation of the coupled voltages at nodes 1 and 2 (Fig. 1) are computed for variables $R_{1}, R_{2}$, and $\theta$, which are uniformly distributed in ranges $[48-52] \Omega, \quad[48-52] \Omega$, and $[110-120]^{\circ}$, respectively, at 17 frequency points equally located between $f=0.9 \mathrm{GHz}$ and $f=2.5 \mathrm{GHz}$. [Figs. 2(a) and (b)]. The accuracy of the Stroud-3 integration rule is demonstrated by calculating the average and standard deviation of the coupled voltages at nodes 1 and 2 at $f=0.9 \mathrm{GHz}$ using a 5 point Gauss-Legendre integration rule. The results are compared in Table I. It should be noted here that the Gauss-Legendre integration rule required 125 deterministic EMI/EMC simulations while Stroud-3 integration rule needed only 6 . The collocation method is embarrassingly parallel; each deterministic EMI/EMC simulation is performed on 16 processors $\left(P_{\mathrm{EM}}=15\right)$.

\section{CONCLUSIONS}

This paper presented a fast and parallel Stroud-based stochastic collocation method for statistically characterizing EMI/EMC phenomena on loaded multiscale platforms with uncertain system configurations and subject to variable electromagnetic excitations. The proposed method uses a previously developed parallel hybrid TDIE-based field-cable- circuit simulator to perform deterministic EMI/EMC simulations. The number of these simulations is only $N+1$ and $2 N$ for Stroud-2 and Stroud-3 integration rules, respectively. Here $N$ is the number of variables being changed in the system configuration and excitation.

\section{REFERENCES}

[1] H. Bagci, A. E. Yilmaz, J. M. Jin, and E. Michielssen, "Fast and rigourous analysis of EMC/EMI phenomena on electrically large and complex cable-loaded structures," IEEE Trans. Electromagn. Compat., vol. 49, no. 2, pp. 361-381, May 2007.

[2] H. Bagci, A. E. Yilmaz, and E. Michielssen, "FFT-accelerated MOTbased solution of time-domain BLT equations," in Proc. IEEE AP-S. Int. Symp., 2006, pp. 1175 - 1179.

[3] H. Bagci, A. E. Yilmaz, and E. Michielssen, "Full TDIE-based modeling of electromagnetic coupling into lossy multiconductor cables on electrically large platforms," in Proc. USNC/URSI National Radio Sci. Meet., 2005.

[4] A. E. Yilmaz, J.-M. Jin, and E. Michielssen, "A parallel FFT accelerated transient field-circuit simulator," IEEE Trans. Microwave Theory Tech., vol. 53, no. 9, pp. 2851-2865, Sept. 2005.

[5] A. E. Yilmaz, J.-M. Jin, and E. Michielssen, "Time domain adaptive integral method for surface integral equations," IEEE Trans. Antennas Propagat., vol. 52, no. 10, pp. 2692-2708, Oct. 2004.

[6] A. E. Yilmaz, D. S. Weile, B. Shanker, J.-M. Jin, and E. Michielssen, "Fast analysis of transient scattering in lossy media," IEEE Antennas Wireless Propagat. Lett., vol. 1, no. 1, pp. 14-17, 2002.

[7] D. Xiu and J. S. Hesthaven, "High-order collocation methods for differential equations with random inputs," J. Sci. Comput., vol. 27, no. 3, pp. 1118-1139, 2005.

[8] A. H. Stroud, "Remarks on the disposition of points in numerical integration formulas 11," Math. Tables Other Aids Comput., vol. 11, no. 60, pp. 257-261, Oct. 1957.

[9] C. R. Paul, Analysis of Multiconductor Transmission Lines. New York: Wiley, 1994. 